\title{
Negative priming depends on ease of selection
}

\author{
ERIC RUTHRUFF \\ University of California, San Diego, La Jolla, California \\ and \\ JEFF MILLER \\ University of Otago, Dunedin, New Zealand
}

\begin{abstract}
Negative priming effects have been offered as evidence that distractor stimuli are identified. We conducted two experiments to determine if such effects occur even when it is easy to discriminate target from distractor stimuli. In Experiment 1, we found the usual negative priming effect when target and distractor positions varied from trial to trial, but not when these positions remained fixed. Experiment 2 extended these results to a situation where the ease of selection varied only in the prime display. These findings argue that irrelevant inputs can be filtered out prior to stimulus identification under certain circumstances and therefore pose problems for strict late selection theories.
\end{abstract}

A central and as yet unresolved issue in the field of selective attention concerns the point at which relevant stimuli begin to be processed more extensively than irrelevant ones. According to strict late selection theories (e.g., Deutsch \& Deutsch, 1963), all stimuli are identified regardless of their relevance to the task at hand, but only a subset of these are selected for input to later processes (e.g., response selection). Early selection theories, on the other hand, assert that unselected stimuli are "filtered out" at an early stage of processing, prior to complete stimulus identification. The strongest early selection theories (e.g., Broadbent, 1958) propose that unselected stimuli are discarded prior to identification; however, in weaker versions of such theories, unselected stimuli are attenuated rather than discarded (e.g., Treisman, 1960). In either case, early selection theories predict that relevant stimuli are more likely to be identified than are irrelevant stimuli (which we will call selective identification), whereas strict late selection theories predict unselective identification.

To determine whether selective identification is possible in selective attention tasks, many researchers have examined the fate of irrelevant stimuli. The results indicate that such stimuli are processed semantically (i.e., are identified) in many experimental settings (e.g., Corteen \& Dunn, 1974; Corteen \& Wood, 1972; Eriksen \& Eriksen,

During the course of this research, the first author received support from a National Science Foundation Graduate Fellowship and Public Health Service Training Grant 2T32-MH14268. The research was also supported by National Institute of Mental Health Grant PHS-40733. We would like to thank Cassandra Fink and Ian Fisher for assistance in testing subjects, and Gordon Logan, Cathleen Moore, Toby Mordkoff, Janice Murray, Hal Pashler, Doug Rohrer, and two anonymous reviewers for useful comments on earlier versions of the manuscript. Correspondence concerning this article should be addressed to E. Ruthruff, Department of Psychology, UCSD, La Jolla, CA 920930109, or to J. Miller, Department of Psychology, University of Otago, P.O. Box 56, Dunedin, New Zealand.
1974; Stroop, 1935). For instance, when subjects are asked to name the color in which a string of letters is printed, naming latencies are very long if the letters happen to spell a conflicting color name (Stroop, 1935). This phenomenon, known as the Stroop effect, suggests that words are identified even when they are not relevant to the current task.

Although the identities of irrelevant stimuli often interfere with the concurrent processing of relevant stimuli, these interference effects have been shown to diminish in experimental conditions that facilitate selection of relevant stimuli. The Stroop effect, for instance, is greatly reduced when the object whose color is to be named is physically separated from the irrelevant color word (Kahneman \& Henik, 1981). Similarly, Yantis and Johnston (1990; but see Miller, 1991) were able to virtually eliminate another type of interference effect by using conditions designed to encourage optimal attentional focusing. These findings support the view that irrelevant objects can be filtered out at an early stage of perception (i.e., selective identification is possible) but that the success of the filter depends upon many factors, including the ease of discriminating relevant from irrelevant stimuli. It should be noted that this view is quite compatible with evidence that subjects can identify multiple objects simultaneously (e.g., Shiffrin \& Gardner, 1972), because the claim that identification can be selective does not imply that it must be selective.

Theorists favoring the view that selection occurs after identification, however, have pointed out that the identification of distractors need not necessarily result in interference effects. Therefore, it is logically possible that irrelevant stimuli are being identified even when interference effects are absent (e.g., Allport, Tipper, \& Chmiel, 1985; Driver \& Tipper, 1989). Allport et al. and others have strengthened this position considerably by showing that a different measure can sometimes demonstrate semantic processing even when irrelevant stimuli produce no interference. 
In one experiment, Allport et al. (1985) asked subjects to name a line drawing of a target object, displayed in red, while ignoring a distractor drawing displayed in green. In a difficult-selection condition, the locations of the target and distractor drawings varied randomly from trial to trial, whereas in an easy-selection condition, the target always appeared at fixation. They found little or no interference in the easy-selection condition-that is, the semantic category of the distractor item had little effect on the time to name a simultaneously presented target. They did find, however, that subjects were slower to name a target picture when it was semantically related to the distractor picture displayed on the preceding trial. To explain this slowing, which was termed negative priming, they suggested that the distractor on the preceding trial was identified and then suppressed by inhibitory mechanisms. So when the subsequent target was semantically related to this distractor, a certain amount of extra time was required to overcome the residual inhibtion. The most important conclusion was that irrelevant pictures were identified, as evidenced by negative priming, even though identification did not result in interference. From this conclusion, Allport et al. argued that previous reports of negligible or reduced interference (e.g., Eriksen \& Eriksen, 1974; Francolini \& Egeth, 1980; Kahneman \& Henik, 1981; Kahneman \& Treisman, 1984) do not unambiguously support early selection. Similar findings of negative priming in conditions producing small or negligible interference effects have since been reported by Driver and Tipper (1989) and Fox $(1994,1995)$.

Negative priming, which has now been observed with a variety of stimuli and tasks (e.g., Dalrymple-Alford \& Budayr, 1966; Neill, Terry, \& Valdes, 1994; Tipper, 1985), is a particularly important source of evidence for nonselective identification because it occurs even when interference is greatly reduced or eliminated. Assuming that the absence of interference implies optimal conditions for selection, the accompanying negative priming appears to indicate that irrelevant stimuli are identified even under optimal selection conditions - that is, that selective identification never occurs. This assumption seems reasonable but might nevertheless be unjustified. Suppose interference effects disappear as soon as selection is easy enough for the early filter to slow identification of irrelevant stimuli. Negative priming effects might still remain under such conditions, because delayed distractor identification could cause negative priming on the next trial even if it were too late to cause much interference on the current trial (Broadbent \& Gathercole, 1990; Driver \& Tipper, 1989). Thus, although negligible interference effects do suggest that selection is relatively easy, they do not necessarily indicate that selection is easy enough for negative priming to disappear.

In fact, each of the previous studies demonstrating negative priming in the absence of significant interference seems to have used conditions that were less than optimal for the efficient selection of relevant stimuli. Driver and Tipper (1989, Experiment 1), for instance, asked subjects to count the number of red items in a display while ignoring the black items. Because both red and black items could appear in any of the eight possible stimulus locations, it seems unlikely that selection was especially easy. Fox $(1994,1995)$ precued target location $150 \mathrm{msec}$ before stimulus onset, but the cue neither significantly reduced overall RT nor completely eliminated distractor-based interference effects, so it probably did not allow efficient selection of the relevant stimulus. Perhaps the most convincing evidence of negative priming in conditions thought to favor efficient selection comes from the easy-selection condition studied by Allport et al. (1985), in which the target always appeared at fixation. In this condition, however, the distractor location varied randomly from trial to trial, and it is possible that the putative early filter can prevent the identification of irrelevant objects much more effectively when the locations of these objects are known in advance. If so, then selection might have been further facilitated by using predictable distractor locations.

In sum, because previous experiments may not have done enough to facilitate selection, and because of the theoretical importance of negative priming effects, the present experiments were designed as stronger tests of the claim that negative priming persists even when selection is easy. If selective identification is possible, then negative priming effects should diminish greatly and perhaps even disappear under more ideal conditions for selection. But if the identification of irrelevant stimuli cannot be prevented, then priming effects (negative or facilitory) should be preserved despite our attempts to create optimal conditions for efficient selection.

\section{EXPERIMENT 1}

The basic design of Experiment 1 replicated Allport et al.'s (1985, Experiments 6-8) manipulation of the uncertainty of the target location and extended this manipulation by including conditions in which selection was made even easier by using fixed distractor locations. In addition, we manipulated the discriminability of the colors in which the target and distractor stimuli were drawn, because it seemed worthwhile to try more than one manipulation of selection ease. At issue in this experiment was whether the amount of negative priming would be sensitive to either or both of these manipulations.

Stimulus displays contained two colored letters: a target and a distractor. The subjects' task was to identify the target letter in each display, designated by its color, by rapidly pressing one of four response keys. A single trial consisted of a prime display followed by a probe display, and the negative priming effect was measured by varying the relationship between the distractor letter in the prime display and the target letter in the probe display. On ignored repetition trials, the target letter in the probe display was the same letter just used as a distractor in the prime display; on control trials, the probe target was different from the prime distractor. Examples of ignored-repetition and control trials are shown in Fig- 


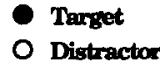

Control

\section{Ignored}

Repetition

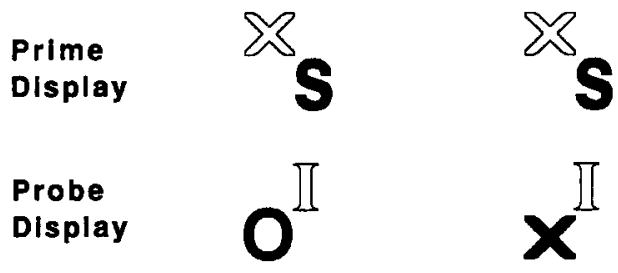

Figure 1. Examples of control and ignored-repetition trials of Experiment 1 . In the actual stimulus displays, target and distractor letters were distinguished by their colors; here, however, targets are shown as filled letters and distractors as unfilled letters.

ure 1. On the basis of previous results (e.g., Allport et al., 1985), responses on ignored-repetition trials were expected to be slower than those on control trials, and the measure of primary interest (i.e., the amount of negative priming) was defined as the difference in probe display reaction time (RT) between the ignored-repetition and control conditions. As is common in these designs, we also included attended-repetition trials, in which the probe target was identical to the prime target.

Following Allport et al. (1985), we manipulated the ease of selection by varying the subject's uncertainty concerning the locations of the target and distractor letters. For one group of subjects, the position of the target and distractor letters varied randomly from trial to trial. This is a replication of the difficult-selection condition of Allport et al. For another group of subjects, the target always appeared at fixation, but the distractor location varied randomly from trial to trial, replicating Allport et al.'s easy-selection condition. A third group of subjects was tested in a previously unexamined condition in which the target and distractor locations remained constant throughout a block of trials. We refer to this as the very-easy-selection condition, because target selection could be even easier than in the easy-selection condition studied by Allport et al., especially if the early filter can more easily prevent the identification of distractors whose locations are known in advance.

\section{Method}

Subjects. Eighty-four undergraduates ( 44 males, 40 females) at the University of California, San Diego, primarily 18-24 years old, participated to fulfill a class requirement. All subjects reported having normal or corrected-to-normal visual acuity.

Stimuli. Stimuli were the uppercase letters S, O, X, and I, chosen because of their high interstimulus discriminability. Letters were created by drawing straight line segments between points in a 5 (horizontal) $\times 7$ (vertical) array. They were displayed on an NEC multi-sync monitor connected to an IBM-PC-compatible computer and subtended approximately $0.6^{\circ}$ horizontally and $1.2^{\circ}$ vertically from a typical viewing distance of $50 \mathrm{~cm}$. Stimuli were displayed in green, purple, white, or light blue, corresponding to EGA/VGA mode palette numbers $10,13,15$, and 11 , respectively.
Procedure. The subjects were instructed to indicate the identity of each target letter by pressing the "n," "m," ",", or "." key with the fingers of their dominant hand. The mapping of the four stimulus letters to the four response keys was randomly selected for each subject. The subjects were told to respond as quickly as possible without making many mistakes. They were also given strict instructions to maintain their gaze at the fixation point throughout each trial.

Targets and distractors were differentiated by their colors. Half of the subjects discriminated between green and purple letters, which were highly discriminable, and the other half discriminated between white and light blue letters, which were less discriminable. Selective identification predicts that the amount of negative priming will be smaller when selection is easy-that is, when the colors are highly discriminable. The assignment of colors to target and distractor stimuli was counterbalanced across subjects.

The ease of selection was also manipulated by varying the location uncertainty of the target and distractor stimuli, as in Allport et al. (1985). There were three levels of location uncertainty, manipulated between subjects. For one third of the subjects, the target and distractor positions varied unpredictably from trial to trial (the difficult-selection condition). In this condition, the target and distractor letters were always positioned along diagonally opposite corners of an imaginary square centered about the fixation point. For another third of the subjects, the target was always presented at fixation, but the distractor appeared randomly in one of four corners of an imaginary square centered about fixation (the easy-selection condition). For the final third of the subjects, the target always appeared at fixation, and the distractor always appeared at the same corner of the imaginary square on every trial within a block (the very-easy-selection condition). ${ }^{1}$ For this group, the distractor position varied randomly between blocks. In order to hold roughly constant the degree of lateral masking between the target and distractor letters, the centers of the target and distractor letters were placed approximately $1.4^{\circ}$ apart in all three selection conditions.

The letters in the prime and probe displays were chosen as follows. On each trial, one of the four stimulus letters was randomly selected to be the target in the probe display, with the restriction that each letter was chosen equally often within a block of trials. The distractor in the probe display was then chosen randomly from the remaining three letters. The letters of the prime display were then selected with the following restrictions, depending upon the experimental condition. In the control condition, the target and distractor stimuli in the prime display were chosen so that they were different from each other and from the letters in the probe display. On the ignoredrepetition trials, the prime distractor was identical to the probe display target, and the prime target was different from the letters in the probe display (see Figure 1). On the attended-repetition trials, the prime target was identical to the probe target, and the prime distractor was different from the letters in the probe display.

Within each block, there were twice as many control trials as ignored-repetition and attended-repetition trials, so that the probability of the prime display target being the same as the probe display target was .25 . Similarly, the probability that the prime distractor would be identical to the probe target was .25 . Note that these are the same probabilities of occurrence as if the four stimulus letters were chosen completely at random.

Each trial began with the presentation of a fixation cross for $700 \mathrm{msec}$ in the center of the display, followed by a blank screen for $300 \mathrm{msec}$. Next, the letters of the prime display were shown for $150 \mathrm{msec}$. Once the subject had responded to the prime display, the fixation cross was displayed again for $200 \mathrm{msec}$. After a blank period of $300 \mathrm{msec}$, the probe letters were displayed for $150 \mathrm{msec}$. If the subject responded incorrectly to the prime and/or the probe, an error message was displayed for $2 \mathrm{sec}$ immediately following the probe response; no feedback was given for correct responses. The next trial began $1.5 \mathrm{sec}$ later.

The subjects completed six blocks of 64 trials in a single session lasting approximately $40 \mathrm{~min}$. The first block was preceded by 32 


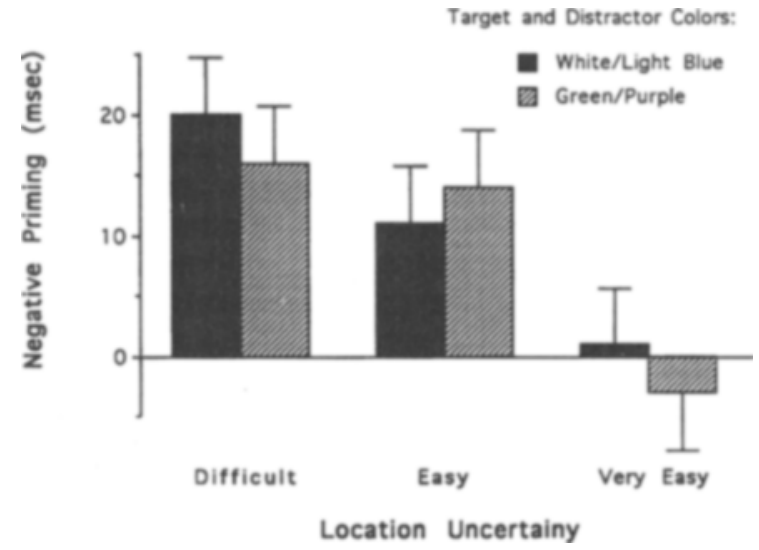

Figure 2. Experiment 1: Mean negative priming effect, with the associated standard error, as a function of location uncertainty and the discriminability of the target and distractor colors.

practice trials, and subsequent blocks were preceded by 3 warmup trials. The subjects were allowed to take a short break at the middle and end of each block.

\section{Results and Discussion}

Trials in which an error was made to the prime or probe display were excluded from analyses of RT, as were trials in which the subject responded in less than $200 \mathrm{msec}$ or more than $1,500 \mathrm{msec}$. The resulting mean RTs to probe displays in the control, ignored-repetition, and attended-repetition trials were 679,689 , and $553 \mathrm{msec}$, respectively, and the corresponding percentages of correct response (PCs) were 96.5, 96.6, and 98.9.

Negative priming. Figure 2 shows the amount of negative priming as a function of location uncertainty and the discriminability of the target and distractor colors. Table 1 shows the mean error rates.

Repeated measures analyses of variance (ANOVAs) were conducted on mean RTs and PCs to the probe display in the ignored-repetition and control conditions. ${ }^{2}$ There was a small but significant negative priming effect on RT: responses were $10 \mathrm{msec}$ slower, on average, in the ignored-repetition condition than in the control condition $[F(1,78)=24.52, p<.001]$. Mean RT also depended on location uncertainty $[F(2,78)=7.26, p<.01]$, with mean RTs of 734, 695, and $624 \mathrm{msec}$ in the difficult-, easy-, and very-easy-selection conditions, respectively. Responses in the very-easy-selection condition were significantly faster than those in the easy-selection condition $(p<.01)$, suggesting that fixing the position of the distractors facilitated selection of the target stimuli. An analysis of error rates revealed no significant effects.

The most important result is that the RT difference between the control and ignored-repetition conditions (i.e., the negative priming effect) depended significantly on location uncertainty $[F(2,78)=7.83, p<.01]$, as shown in Figure 2. In addition, separate analyses within each of the three selection conditions indicated that there was significant negative priming in the difficult- and easyselection conditions $(p<.001)$ but not in the very-easyselection condition $(p>.20)$. Furthermore, the amount of negative priming in the very-easy-selection condition was significantly less than that of the easy-selection condition $(p<.05)$ and that of the difficult-selection condition $(p<$ .05 ). The comparison between the easy- and very-easyselection conditions is especially important because the displays in these two conditions were identical except for the fact that, in the very-easy-selection condition, the subjects could anticipate the location of the distractor letter. Thus, the negative priming effect was reduced or eliminated when selection was especially easy, as predicted by the hypothesis of selective identification.

An analysis including both easy- and difficult-selection conditions, but excluding the very-easy-selection condition, revealed a significant negative priming effect $(p<$ .001 ) that did not vary significantly between the two selection conditions $[F(1,52)=1.53, p>.2]$. Thus, the results of Experiment 1 replicate the results from the difficult- and easy-selection conditions of Allport et al. (1985). It is the new condition, with fixed distractor locations, that seems primarily responsible for the decrease in the negative priming effect. This suggests that advance knowledge of distractor location is important, which is consistent with models in which selective attention operates largely by preventing the identification of distractors-rather than simply enhancing the identification of targets (cf. Shiu \& Pashler, 1994)-and in which filtering is more effective when distractor locations are known in advance.

The negative priming effect did not depend on the discriminability of the target and distractor colors $[F(1,78)<1]$. This result cannot be interpreted as evidence that negative priming is independent of selection ease, however, because color discriminability did not produce a significant main effect.

Facilitation. Similar ANOVAs were conducted on mean probe RTs and error rates in the control and attendedrepetition conditions. As expected, the subjects were faster $[F(1,78)=404.57, p<.001]$ and more accurate $[F(1,78)=$ $84.32, p<.001]$ in the attended-repetition condition than in

Table 1

Mean Error Rates for Experiment 1 as a Function of Trial Type, Location Uncertainty, and Color Discriminability

\begin{tabular}{|c|c|c|c|c|c|c|}
\hline \multirow[b]{3}{*}{ Trial Type } & \multicolumn{6}{|c|}{ Color Discrimination } \\
\hline & \multicolumn{2}{|c|}{ Location Difficult } & \multicolumn{2}{|c|}{ Location Easy } & \multicolumn{2}{|c|}{ Location Very Easy } \\
\hline & Difficult & Easy & Difficult & Easy & Difficult & Easy \\
\hline Attended repetition & 0.7 & 1.6 & 0.8 & 0.6 & 1.9 & 1.0 \\
\hline Ignored repetition & 2.5 & 3.2 & 3.0 & 3.0 & 5.2 & 3.6 \\
\hline Control & 3.2 & 3.1 & 3.4 & 2.8 & 4.9 & 3.8 \\
\hline
\end{tabular}


the control condition. Unlike the negative priming effect, however, this difference did not depend on location uncertainty $[F(2,78)<1]$, nor did it depend on color discriminability $[F(1,78)=1.14, p>.2]$.

Prime displays. Mean prime display RT depended on location uncertainty $[F(2,78)=6.65, p<.01]$; mean RTs were 727,691 , and $627 \mathrm{msec}$ in the difficult-, easy-, and very-easy-selection conditions, respectively. As was the case for probe displays, mean RTs in the very-easyselection condition were significantly faster than those in the easy-selection condition $(p<.05)$.

Interference? The lack of negative priming in the very-easy-selection condition suggests that distractor letters were not identified. If this is the case, then one would certainly expect interference effects to disappear as well. The present design did not allow us to measure interference effects, however, so we tested this prediction by running a control experiment with 48 new subjects equally divided between the difficult- and veryeasy-selection conditions. The stimulus set in the control experiment included an additional letter that sometimes appeared as the distractor but never as the target. Because this neutral stimulus was not associated with a particular response, it should produce less interference, if identified, than would the other letters in the stimulus set (cf. Eriksen \& Eriksen, 1974). Thus, if distractor letters are identified, we expected faster responses to targets accompanied by the neutral distractor than to targets accompanied by one of the other responseassigned letters as a distractor. This effect was obtained with difficult selection $(35 \pm 11 \mathrm{msec}),{ }^{3}$ but not with very easy selection $(1 \pm 6 \mathrm{msec})$. In the very-easyselection condition, then, distractors produced neither negative priming nor interference. Although it is still logically possible that the distractors are nonetheless identified in this condition, as would be revealed by some other measure, the most parsimonious explanation of these results is simply that the distractors were not identified. Thus, the overall conclusion from Experiment 1 is that selective identification is possible, at least under suitable conditions.

\section{EXPERIMENT 2}

The results of Experiment 1 indicate that the amount of negative priming is reduced or even eliminated when it is especially easy to discriminate targets from distractors, contrary to the conclusion of Allport et al. (1985). These results are important because they suggest that the identification of irrelevant stimuli can be prevented under these conditions.

However, one might criticize Experiment 1 because the difficult-, easy-, and very-easy-selection conditions differed not only in the ease with which the target could be selected in the prime display but also in the ease with which the target could be selected on the probe display. It is conceivable that the lack of negative priming in the very-easy-selection condition of Experiment 1 was due not to successful filtering of the prime distractor but to the ease of selection in the probe display. According to this explanation, distractor letters were identified and inhibited in all conditions, but, for some reason, this inhibition only produced negative priming when selection of the probe target was relatively difficult.

There is, in fact, some evidence that the magnitude of negative priming depends on characteristics of the probe display. Allport et al. (1985, Experiment 9), for example, found that negative priming was observed only when the probe display contained a distractor stimulus. Moore (1994) extended this finding by demonstrating that negative priming is generally not observed whenever it can be easily determined that the probe display contains no conflicting information. So, although displays in the very-easy-selection condition of Experiment 1 did contain distractors, it is still conceivable that negative priming was eliminated because they were easy to discriminate from targets.

The purpose of Experiment 2, therefore, was to replicate Experiment 1 using a design where the ease of target selection on the probe display was held constant. Once this was done, any differences across conditions could be attributed unambiguously to differential processing of prime displays.

In both prime and probe displays, stimulus letters $(E, F$, $\mathrm{G}$, and $\mathrm{H}$ ) were drawn by removing line segments from figure eights (Yantis \& Jonides, 1984). The subjects were told to respond on the basis of the identity of the purple letter, while ignoring the green letter. To control the ease of selection on prime trials, we varied the stimulus onset asynchrony (SOA) of the color and identity information. In a "simultaneous" condition ( $\mathrm{SOA}=0 \mathrm{msec}$ ), the two types of information were presented together (i.e., gray figure eights turned into colored letters). In three "identity-first" conditions, the figure eights turned into gray letters, which then changed to purple and green after SOAs of 100,200, or $300 \mathrm{msec}$. In three "color-first" conditions, the gray figure eights changed to purple and green 100,200 , or $300 \mathrm{msec}$ before the letters were revealed. Because it was possible for the subjects to move their eyes before the letters were revealed at the longest SOAs in the color-first condition, the stimuli were presented at approximately the same locations (i.e., overlapping) so that eye movements would not be particularly useful.

When the colors are presented before the letters, stimulus selection should be relatively easy, because the subject can "focus on" the location of the purple figure eight and ignore the green figure eight. Conversely, selection should be difficult when the colors are presented after the letters, because the subject initially does not know which letter identity is the target. To the extent that selective identification is possible when it is easy to select target stimuli, we should observe the greatest negative priming effects in the identity-first conditions and the smallest negative priming effects in the color-first conditions. Alternatively, if selective identification is impossible, then distractors will always be identified, and priming should be substantial regardless of the order in which color and letter information are presented. 
In summary, whereas ease of selection was manipulated in both prime and probe displays in Experiment 1, ease of selection was manipulated only on the prime displays in Experiment 2. This was accomplished by reliably and accurately precuing the locations of the prime display targets and distractors on a proportion of the trials (i.e., in the color-first conditions). Note that because both target and distractor locations were precued, this condition most resembles the very-easy-selection condition of Experiment 1, in which negative priming was virtually eliminated. Because ease of selection on the probe display was held constant across conditions, elimination of negative priming in the precued conditions of Experiment 2 could be confidently attributed to differential processing of distractors (i.e., selective identification) on the prime display.

\section{Method}

Except where noted, the methods were identical to those of Experiment 1 .

Subjects. Thirty undergraduates ( 17 males, 13 females) at the University of California, San Diego, primarily 18-24 years old, participated to fulfill a class requirement. All subjects reported having normal or corrected-to-normal visual acuity. None had participated in Experiment 1

Stimuli. Stimuli were the letters E, F, G, and H, drawn with straight line segments. The letters subtended approximately $0.6^{\circ}$ horizontally and $1.2^{\circ}$ vertically, and their centers were placed approximately $0.6^{\circ}$ apart so that the letters were largely overlapping, with one letter centered above and to the left of the other. Stimuli were displayed in gray, green, or purple, corresponding to EGA/VGA mode palette numbers 7,10 , and 13 , respectively.

Procedure. The subjects were instructed to respond to the purple letters in the prime and probe displays, while ignoring the green letters. They responded to the letters $\mathrm{E}, \mathrm{F}, \mathrm{G}$, and $\mathrm{H}$ by pressing the " $\mathrm{d}$," "c," "m," and " $k$ " keys, respectively, using the middle and index fingers of their left and right hands.

Each trial began with the presentation of the figure eights for $1 \mathrm{sec}$, at which point line segments were removed to reveal the target and distractor letters of the prime display. The figure eights were initially gray on all trials. In the simultaneous condition, the gray figure eights changed abruptly to purple and green letters, exactly $1 \mathrm{sec}$ after their onsets. In the color-first conditions, the gray figure eights corresponding to the target and distractor locations turned purple and green, respectively, 700,800 , or $900 \mathrm{msec}$ after their onsets (i.e., 300,200 , or $100 \mathrm{msec}$ before the letters were revealed). In the identity-first conditions, colors were not presented until 100 , 200 , or $300 \mathrm{msec}$ after the figure eights changed to letters. The SOA for each trial was chosen randomly, with the restriction that each of the seven different SOA conditions occur equally often within a block of trials. The interval between onset of the figure eights and onset of the letters was held constant at 1 sec across all SOA conditions, so that the temporal uncertainty of the to-be-reported information (i.e., letter identity) would be equal across conditions.

Prime letters remained on the screen until the subject made a response. After a $200-m s e c$ blank interval, the gray figure eights were presented again. This time, they remained for $300 \mathrm{msec}$, at which point they abruptly changed into the purple and green letters of the probe display. Thus, the SOA between color and identity information in the probe displays was always 0 msec. The probe display remained visible until the subject responded. If the subject responded incorrectly to either the prime or the probe, an error message was displayed for $800 \mathrm{msec}$ immediately following the probe response; no feedback was given following correct responses. The next trial began $1.5 \mathrm{sec}$ later.
The subjects completed eight blocks of 56 trials in a single session. The first block was preceded by 12 practice trials, and subsequent blocks were preceded by 2 warm-up trials. The entire session lasted approximately $45 \mathrm{~min}$.

\section{Results and Discussion}

Mean RTs to probe displays in the control, ignoredrepetition, and attended-repetition trials were 748,754 , and $610 \mathrm{msec}$, respectively, and the corresponding PCs were $97.2,96.5$, and 99.2 .

Negative priming. Figure 3 shows the mean probe display RTs and error rates in the ignored-repetition and control conditions as a function of SOA. Analyses of probe display RT and PC as a function of SOA and ignoredrepetition versus control conditions yielded a significant overall negative priming effect on PC $[F(1,29)=4.87$, $p<.05]$, but not on RT $[F(1,29)=2.04, p>.1]$.

Although the overall negative priming effect on RT failed to reach significance, the amount of negative priming decreased ( $p<.05$, linear trend) as the SOA between color and identity information decreased - that is, negative priming decreased with increasing ease of selection. In addition, a planned comparison of mean RTs from the two most extreme SOAs (300 msec identity-first, and $300 \mathrm{msec}$ color-first) revealed a significant difference in the amount of negative priming $[F(1,29)=4.25, p<$ .05]. Furthermore, examination of mean RTs at each SOA separately indicated that the negative priming effect was significant at an SOA of $300 \mathrm{msec}$ in the identityfirst condition $[F(1,29)=8.01, p<.01]$, but not at any of the other SOAs $(p>.1)$. Overall, these results are compatible with the claim that negative priming decreases with increasing ease of selection and that negative priming essentially disappears when it is especially easy to discriminate targets from distractors. Similar analyses on $\mathrm{PC}$ indicated a significant effect of negative priming on accuracy only at an SOA of $100 \mathrm{msec}$ in the identity-first condition $[F(1,29)=5.64, p<.05]$; the overall decrease in negative priming with increasing ease of selection was not reliable in the PC data $(p>.10$, linear trend).

The overall effect of negative priming on RT was very weak, except in the identity-first condition at an SOA of $300 \mathrm{msec}$. This was somewhat surprising, because previously published experiments using the simultaneous condition ( 0 -msec SOA) have generally revealed significant negative priming effects on $\mathrm{RT},{ }^{4}$ and, if anything, the effects should be even larger with letters presented first by 100 or $200 \mathrm{msec}$. This finding suggests that some aspect of the present procedure was especially effective at inducing selective identification. The most obvious candidate is the temporal separation of color and identity information in this experiment, because, in most previous experiments, target/distractor and identity information were given simultaneously. Because the two types of information were separated here, and the SOA varied randomly from trial to trial, the subjects in our experiment may have adopted a strategy of waiting for color information to arrive before fully processing letter identities, just as they seem to wait to find out which letter is rele- 

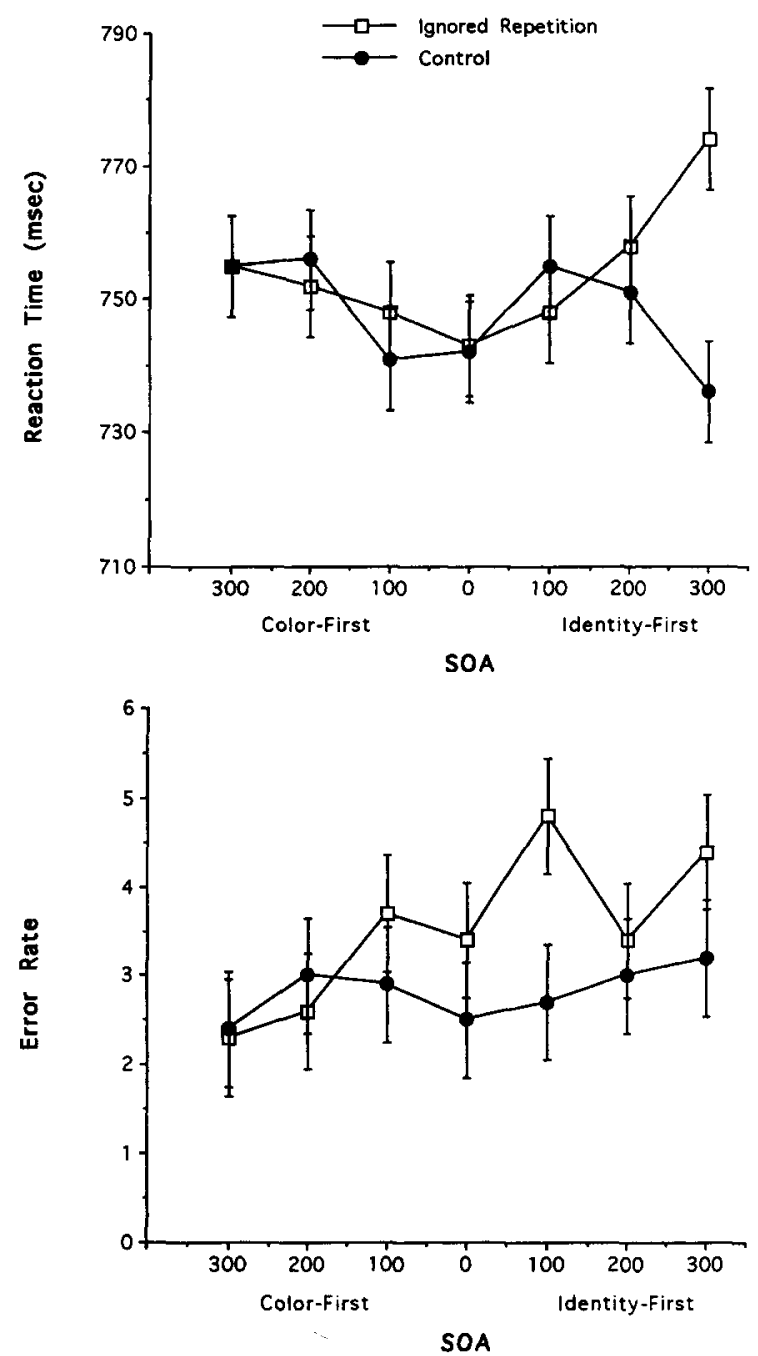

Figure 3. Experiment 2: Ignored-repetition and control condition mean reaction time and error rate as a function of the stimulus onset asynchmony (SOA) between color and identity information. The standard errors about these means were computed using the condition (ignored repetition versus control) $\times \mathrm{SOA} \times$ subjects interaction term as the error term.

vant before fully identifying perceptually degraded letters in a bar-probe task (Mewhort, Johns, \& Coble, 1991; Pashler, 1984). Note that the strategy of preventing the identification of stimulus letters that are not known to be relevant is perfectly consistent with the notion of selective identification. On this view, the presence of negative priming at the longest SOA in the identity-first condition might reflect an inability, or an unwillingness, to indefinitely defer identification of all stimuli.

Another possibility, suggested by a comparison of the present procedure with that of Experiment 1 , is that negative priming was diminished because stimulus letters were drawn by removing segments from figure eights. It might be easier to avoid identifying offset stimuli than onset stimuli (Yantis \& Jonides, 1984).
To check on the importance of both temporal separation and the use of figure eights, we ran a control experiment $(N=23)$ in which color and identity information were always presented simultaneously on both prime and probe displays. On half of the trials, the letters were revealed by removing line segments from the figure eights (exactly as in the main experiment); on the other half of the trials, the letters appeared abruptly, without figure eights. This control experiment revealed a reliable $(p<.001)$ negative priming effect of $20 \pm 10 \mathrm{msec}$. Contrary to the hypothesis that figure eights prevent negative priming, we actually observed somewhat greater negative priming with figure eights $(M=23 \mathrm{msec})$ than without ( $M=17 \mathrm{msec})$, although this difference did not approach significance $(p>.10)$. Thus, we conclude that the lack of negative priming in the main experiment at the $0-\mathrm{msec}$ SOA likely resulted from the temporal separation of color and identity information, rather than the use of figure eights.

Another control experiment $(N=10)$ was run using only simultaneous and identity-first SOAs (100 and $200 \mathrm{msec}$ ), and these SOAs yielded reliable negative priming effects $(p<.05)$ of 37,39 , and $48 \mathrm{msec}$, respectively. Thus, the extent of negative priming in the identity-first conditions seems to depend on whether color-first conditions are also tested in the experiment, which provides additional evidence that selective or nonselective identification may be partly strategic.

One possible explanation for these strategic effects is that when color information often arrives prior to the onset of the letters (as in Experiment 2), subjects adopt the strategy of deferring identification until the relevant object can be selected. But when color information is never presented prior to stimulus onset (i.e., there are no colorfirst conditions, as in the previously discussed control experiments), then color information will never arrive before the onset of the letters. Rather than always defer identification of the letters until the color information arrives, subjects choose to begin processing letter identities immediately. This hypothesis correctly predicts that the negative priming resulting from distractors in the simultaneous and identity-first conditions should be greatly reduced whenever color-first conditions are also used in the experiment. Because letter identification tends to be triggered by the onset of color information in these conditions, this hypothesis also predicts that once color information arrives, it should make little difference how much earlier the letters were presented. As discussed below, the prime data support this prediction.

Facilitation. ANOVAs were conducted on mean probe display RTs and PCs in the control and attendedrepetition conditions. As expected, the subjects were much faster $[F(1,29)=231.97, p<.001]$ and more accurate $[F(1,29)=34.05, p<.001]$ in the attendedrepetition condition. There was also a significant main effect of $\operatorname{SOA}[F(6,174)=2.47, p<.05]$; the subjects responded slightly faster to probe displays following color-first prime displays. 
Table 2

Mean Reaction Time to Prime Displays in Experiment 2 as a Function of SOA

$$
\text { SOA }
$$

\begin{tabular}{lcccccccc}
\cline { 2 - 6 } & \multicolumn{3}{c}{ Color-First } & & \multicolumn{3}{c}{ Identity-First } \\
\cline { 2 - 6 } \cline { 7 - 9 } & 300 & 200 & 100 & 0 & & 100 & 200 & 300 \\
\hline Reaction time & 762 & 770 & 778 & 836 & 799 & 789 & 799 \\
Error rate & 1.4 & 1.4 & 2.6 & 2.2 & 2.9 & 2.4 & 3.2 \\
\hline
\end{tabular}

Prime displays. Mean error rates and RTs to the prime displays, measured from the moment both color and identity information were available for processing, are given in Table 2 as a function of SOA. The effect of SOA on RT was significant overall $[F(6,174)=17.18$, $p<.001]$. In addition, the simultaneous condition was reliably slower $(p<.05)$ than each of the other conditions, indicating that subjects are helped by a preview of either color or identity information (i.e., information available before the RT clock starts). Furthermore, for every SOA, the RT benefit of color preview was reliably larger than that of an identity preview of the same duration $(p<.05)$, indicating that it is especially helpful to know the target and distractor locations in advance of their identities. Because of the relatively small benefit of identity preview, it seems unlikely that the subjects took advantage of the preview by fully processing letter identities (cf. Pashler, 1984).

\section{GENERAL DISCUSSION}

The results of the present experiments indicate that negative priming is reduced in conditions that facilitate selection and, in fact, can disappear completely when selection is especially easy. In Experiment 1, we found reliable negative priming effects when both target and distractor positions varied from trial to trial (the difficultselection condition) and when only the distractor position varied from trial to trial (the easy-selection condition), but there was no evidence of negative priming when both target and distractor positions were fixed throughout a block of trials (the very-easy-selection condition). The latter finding, in combination with the absence of interference effects, suggests that distractor stimuli were not identified on prime trials when selection was sufficiently easy, as predicted by the hypothesis that selection can occur prior to identification. Experiment 2 extended the results of Experiment 1 to a situation in which ease of selection varied only in the prime display, eliminating alternative explanations in which the absence of negative priming is attributed to the ease of selecting the target in the probe display.

The lack of negative priming argues that irrelevant inputs can sometimes be filtered out prior to stimulus identification and therefore poses a problem for strict late selection theories. It appears that distractors are identified, resulting in interference, negative priming, or both, only when filtering has not been completely successful. Naturally, when the filter does fail, a late (i.e., postidentification) selection mechanism-perhaps involving inhibitory mechanisms-may be used to prevent responses to distractors.

The present conclusion contradicts several previous reports that negative priming does not decrease with increasing ease of selection (e.g., Allport et al. 1985; Driver \& Tipper, 1989; Fox, 1994, 1995). Perhaps the starkest empirical contradiction concerning negative priming is between the results of the present Experiment 2 and those of Fox $(1994,1995)$, who used a similar paradigm to study the effects of selection ease on negative priming. She presented a precue at or near the location of the target letter $150 \mathrm{msec}$ before that letter appeared, or no precue at all, and measured both negative priming and interference effects produced by distractors. Although interference was reduced by precues, negative priming was at least as large with them as without them, suggesting that distractor letters are still identified even when target location is cued in advance. The effectiveness of Fox's precues can be questioned, however, because (1) the main effect of cuing on RT was small and not statistically reliable, and (2) cuing did not entirely eliminate distractor-based interference. Thus, it is doubtful that Fox's cues were as effective as possible at encouraging selection, and a simple reconciliation with the present Experiment 2 is that our precues were more effective. The failures to observe decreased negative priming under conditions thought to facilitate selection reported by Allport et al. (1985) and Driver and Tipper (1989) can also be criticized on the grounds that conditions were not truly optimal for efficient selection of target stimuli. Moreover, Kramer, Humphrey, Larish, Logan, and Strayer (1994) have also found that negative priming is greatly reduced when target location is cued in advance.

The present results, however, do not contradict previous findings that distractors can produce negative priming even when they do not produce much interference (e.g., Allport et al., 1985; Driver \& Tipper, 1989; Fox, 1994, 1995). One possible explanation of this apparent dissociation is that, under some conditions, distractors are attenuated effectively enough to slow their rate of identification relative to that of target stimuli. They would thus be identified too late to produce much interference on the current trial, yet soon enough to produce negative priming on the next trial (Broadbent \& Gathercole, 1990). This explanation can be extended quite naturally to account for the disappearance of negative priming in the very-easyselection condition, by assuming that this condition allows even greater distractor attenuation.

One might attempt to preserve the idea that selection occurs subsequent to identification by arguing that irrelevant stimuli are always identified, but, for some extraneous reason, they sometimes fail to cause interference and negative priming when selection is very easy. This possibility cannot be denied, but it does not seem very likely. If a distractor is fully identified, and the same letter reappears as the target $0.5 \mathrm{sec}$ later, one would expect either especially slow responses (i.e., negative priming, as is typically found) or very fast responses 
(facilitory priming) to the latter target. Specifically, negative priming would be expected if the initial distractor representation were inhibited in some fashion (e.g., Allport et al., 1985), and facilitory priming would be expected if it were not (e.g., Allport et al., 1985, Experiments 3-4; Posner, 1978). Yet we observed essentially no priming of either type in the present experiments when selection was very easy. It is still logically possible that distractors were identified and that the resulting facilitation and inhibition happened to cancel each other out. Rather than invoke such a coincidence to explain the results, however, it seems more parsimonious at the present time to conclude that distractors were simply not identified when selection was very easy.

Although the present results support claims that early filtering of irrelevant inputs is possible, they also provide further evidence that the filter is not always perfectly successful (e.g., in the easy-selection condition of Experiment 1). Thus, it remains a possibility that, much of the time, attentional selection will occur after identification-that is, "late." Even in this case, however, early filtering might play an important role by reducing the activations of irrelevant stimuli, thus making it easier for late selection mechanisms to exclude them.

\section{REFERENCES}

Allport, D. A., TipPer, S. P., \& Chmiel, N. R. J. (1985). Perceptual integration and postcategorical filtering. In M. I. Posner \& O. S. M. Marin (Eds.), Attention and performance XI (pp. 107-132). Hillsdale, NJ: Erlbaum.

Broadbent, D. E. (1958). Perception and communication. London: Pergamon Press.

BroadBent, D. E., \& Gathercole, S. E. (1990). The processing of nontarget words: Semantic or not? Quarterly Journal of Experimental Psychology, 42A, 3-37.

CorTeEn, R. S., \& DunN, D. (1974). Shock-associated words in a nonattended message: A test for momentary awareness. Journal of Experimental Psychology, 102, 1143-1144.

CORTEEN, R. S., \& Woon, B. (1972). Autonomic responses to shock associated words in an unattended channel. Journal of Experimental Psychology, 97, 308-313.

DALRYMPLE-AlFoRD, E. C., \& BUdAyr, B. (1966). Examination of some aspects of the Stroop colour-word test. Perceptual \& Motor Skills, 23, 1211-1214.

Deutsch, J. A., \& Deutsch, D. (1963). Attention: Some theoretical considerations. Psychological Review, 70, 80-90.

DRIVER, J., \& TIPPER, S. P. (1989). On the nonselectivity of "selective" seeing: Contrasts between interference and priming in selective attention. Journal of Experimental Psychology: Human Perception \& Performance, 15, 304-314.

ERIKSEN, B. A., \& ERIKSEN, C. W. (1974). Effects of noise letters upon the identification of a target letter in a nonsearch task. Perception \& Psychophysics, 16, 143-149.

Fox, E. (1994). Interference and negative priming from ignored distractors: The role of selection difficulty. Perception \& Psychophysics, 56, 565-574.

Fox, E. (1995). Precueing target location reduces interference but not negative priming from visual distractors. Quarterly Journal of Experimental Psychology, 48A, 26-40.

Francolini, C. M., \& EGETH, H. E. (1980). On the nonautomaticity of "automatic" activation: Evidence of selective seeing. Perception \& Psychophysics, 27, 331-342.

GreEnhouse, S. W., \& GersSER, S. (1959). On methods in the analysis of profile data. Psychometrika, 24, 95-112.

Kahneman, D., \& HeniK, A. (1981). Perceptual organization and at- tention. In M. Kubovy \& J. R. Pomerantz (Eds.), Perceptual organization (pp. 181-211). Hillsdale, NJ: Erlbaum.

Kahneman, D., \& Treisman, A. M. (1984). Changing views of attention and automaticity. In R. Parasuraman \& D. R. Davies (Eds.), $\mathrm{Va}$ rieties of attention (pp. 29-61). New York: Academic Press.

KRAMER, A. F., Humphrey, D. G., LARISH, J. F., LOGAN, G. D., \& STRAyer, D. L. (1994). Aging and inhibition: Beyond a unitary view of inhibitory processing in attention. Psychology \& Aging, 9, 491-512.

MewhorT, D. J. K., Johns, E. E., \& CoBle, S. (1991). Early and late selection in partial report: Evidence from degraded displays. Perception \& Psychophysics, 50, 258-266.

MilleR, J. O. (1991). The flanker compatibility effect as a function of visual angle, attentional focus, visual transients, and perceptual load: A search for boundary conditions. Perception \& Psychophysics, 49, 270-288.

Moore, C. M. (1994). Negative priming depends on probe-trial conflict: Where has all the inhibition gone? Perception \& Psychophysics, 56, 133-147.

NeILL, W. T., TerRY, K. M., \& VALDES, L. A. (1994). Negative priming without probe selection. Psychonomic Bulletin \& Review, 1, 119-121

PASHLER, H. E. (1984). Evidence against late selection: Stimulus quality effects in previewed displays. Journal of Experimental Psychology: Human Perception \& Performance, 10, 429-448.

PosNer, M. I. (1978). Chronometric explorations of mind. Hillsdale, $\mathrm{NJ}$ : Erlbaum.

ShIFFrin, R. M., \& GARDNER, G. T. (1972). Visual processing capacity and attentional control. Journal of Experimental Psychology, 93, 72-82.

SHIU, L.-P., \& PASHLER, H. E. (1994). Negligible effects of spatial precuing on identification of single digits. Journal of Experimental $P$ sychology: Human Perception \& Performance, 20, 1037-1054.

STROOP, J. R. (1935). Studies of interference in serial verbal reactions Journal of Experimental Psychology, 18, 643-662.

TiPPER, S. P. (1985). The negative priming effect: Inhibitory priming by ignored objects. Quarterly Journal of Experimental Psychology, 37A, 571-590.

Treisman, A. M. (1960). Contextual cues in selective listening. Quarterly Journal of Experimental Psychology, 12, 242-248.

YANTIS, S., \& JoHNSTON, J. C. (1990). On the locus of visual selection: Evidence from focussed attention tasks. Journal of Experimental Psychology: Human Perception \& Performance, 16, 135-149.

YANTIS, S., \& JoNIDES, J. (1984). Abrupt visual onsets and selective attention: Evidence from visual search. Journal of Experimental Psychology: Human Perception \& Performance, 10, 601-621.

\section{NOTES}

1. Because subjects knew where the target and distractor letters would appear, it would have been possible for them to fixate to the side of the target letter away from the distractor, so that the image of the distractor letter would fall farther into the periphery of the retina. Of course, this would also place the target farther into the periphery, so it is not clear how much could be gained from such a strategy. In any case, this was not possible in the other conditions, and so would represent a possible confound. To prevent subjects from using this strategy, they were given strict instructions to always maintain fixation at the center of the screen, and no subject reported failures to do so during the postexperiment debriefing.

2 . In all of the analyses reported in this article, $p$ values have been adjusted using Greenhouse and Geisser's (1959) correction where appropriate. Comparable analyses have also been conducted using individual subject median RTs, and these yielded similar results.

3. All confidence intervals reported in this article are $95 \%$ confidence intervals.

4. On the other hand, it is quite possible that failures to observe negative priming are equally frequent but tend not to be reported due in part to a general bias against publishing nonsignificant results.

(Manuscript received September 6, 1994; revision accepted for publication December 12, 1994.) 